\title{
L'adoption du Contrôle de Gestion à l'Hôpital Marocain : Explication par l'approche PLS
}

\author{
Boussetta Mohamed, (PhD) \\ Alami Saad, (PhD Student)
}

Université Mohammed V de Rabat

Faculté des Sciences Juridiques, Economiques et Sociales, Agdal, Maroc

doi: 10.19044/esj.2017.v13n4p167 URL:http://dx.doi.org/10.19044/esj.2017.v13n4p167

\begin{abstract}
For many authors the adoption of management control by companies is justified by the positive correlation between the implementation of this system and the increase of the organizational performance of the company. In Morocco, a single study focused on understanding the determinants of the choice of implementation of management control in the Moroccan context (Ahsina K., 2012), that is why we had the idea of developing a conceptual model adapted to Moroccan public hospitals.

As a result, our problem is as follows: What is the impact of the determinants of management control on the organizational performance of the Moroccan public hospital ?

This article presents, in a first point a review of the literature and the formulation of research hypotheses, in a second point the research methodology and in a last point the results of the descriptive analysis of the research.
\end{abstract}

Keywords: Management Control, Moroccan public hospital, Partial Least Squares

Résumé:

Pour plusieurs auteurs l'adoption du contrôle de gestion par les entreprises est justifiée par la corrélation positive entre la mise en place de ce système et l'augmentation de la performance organisationnelle de l'entreprise.

$\mathrm{Au}$ Maroc, une seule étude s'est intéressée à la compréhension des déterminants des choix de la mise en place du contrôle de gestion dans le contexte Marocain (Ahsina K., 2012), c'est pourquoi nous avons eu l'idée de développer un modèle conceptuel adapté aux hôpitaux publics Marocains. 
De ce fait, notre problématique est comme suit : Quel est l'impact des déterminants du contrôle de gestion sur la performance organisationnelle de l’hôpital public Marocain ?

Cet article présente, dans un premier point une revue de la littérature et la formulation des hypothèses de recherche, dans un second point la méthodologie de recherche et dans un dernier point les résultats de l'analyse descriptive de la recherche.

Mots clés: Contrôle de gestion, hôpital public Marocain, Partial Least Squares

\section{Introduction :}

L’importance du contrôle de gestion dans la recherche de la performance organisationnelle, se situe au tout premier plan des débats actuels. Certes, il veille à la déclinaison de la stratégie et le pilotage de la performance globale, mais il a aussi un autre rôle plus interactif et stratégique. Pour plusieurs auteurs l'adoption du contrôle de gestion par les entreprises est justifiée par la corrélation positive entre la mise en place de ce système et l'augmentation de la performance organisationnelle de l'entreprise.

Aujourd'hui personne ne peut nier cette importance, mais malheureusement son adoption est entravée surtout dans le secteur public par plusieurs raisons.

Au Maroc, une seule étude s’est intéressée à la compréhension des déterminants des choix de la mise en place du contrôle de gestion dans le contexte Marocain (Ahsina K., 2012), c'est pourquoi nous avons eu l'idée de développer un modèle conceptuel adapté aux hôpitaux publics Marocains.

De ce fait, notre problématique est comme suit : Quel est l'impact des déterminants du contrôle de gestion sur la performance organisationnelle de l'hôpital public Marocain?

Cet article présente, dans un premier point une revue de la littérature et la formulation des hypothèses de recherche, dans un second point la méthodologie de recherche et dans un dernier point les résultats de l'analyse descriptive de la recherche.

\section{Revue de la littérature et présentation des hypothèses de recherche :}

La proposition du modèle théorique de l'adoption du contrôle de gestion et de son impact sur la performance organisationnelle nécessite un survol sur la littérature consacrée à ce concept.

Depuis Anthony R.N. (1965), plusieurs définitions et typologies du contrôle de gestion ont été proposées. " Le contrôle de gestion est le 
processus par lequel les managers influencent d'autres membres de l'organisation pour mettre en œuvre les stratégies de l'organisation » (Anthony, 1988). En effet, de l'évolution des conceptions du contrôle de gestion, on constate que ce dernier joue un rôle beaucoup plus actif quant à la réalisation de la stratégie définie par l’organisation.

La théorie de la contingence a mis en valeur l'aspect « adaptable » du contrôle de gestion aux facteurs contextuels des organisations. C'était le passage d'une approche classique censée être universelle applicable à toutes les organisations à une approche plutôt flexible qui s'adapte aux exigences du contexte » (Ahsina K., Taouab O. et Nafzaoui M.A., 2014).

\subsection{La taille de l'organisation et le contrôle de gestion}

Parmi les facteurs de contingence on trouve la taille de l'organisation (Dupuy, 1990 ; Mintzberg, 2006), qui est corrélée avec sa propension à connaître et à adopter les outils du contrôle de gestion. Elle occupe une place centrale parmi les variables contextuelles qui sont associées à l'adoption des outils du contrôle de gestion. Dahlgren et alii. (2001) ont démontré que le facteur taille est significatif pour expliquer l'adoption du contrôle de gestion. Les techniques budgétaires sont d'autant plus sophistiquées que la taille des organisations est importante (Merchant, 1981 ; Kalika, 1987). Elle exerce une influence sur le contenu des outils de contrôle de gestion. Les indicateurs de performance non financiers sont plus utilisés par les grandes organisations que par celles de taille moyenne. Nobre (2001) confirme ce point et indique que les organisations de moins de 100 salariés utilisent peu ou pas de tableaux de bord intégrant des mesures physiques. Il précise que la taille de l'organisation constitue un facteur de contingence explicatif des pratiques de pilotage des organisations. Les représentations formelles non financières de la performance sont à priori plus répandues dans les grandes organisations que dans celles de taille moyenne.

De ces travaux, nous formulons l'hypothèse suivante :

Hypothèse 1 : "Plus la taille de l'hôpital est grande, plus le contrôle de gestion est susceptible d'émerger et de se développer ".

\subsection{La planification stratégique et la mise en place du contrôle de gestion}

Au-delà du choix d'une stratégie d'activité, les dirigeants doivent opter pour une stratégie de contrôle. Durant les années soixante-dix et quatre-vingts les travaux de Miles et Snow (1978) et ceux de Porter (1985) ont tenté de démontrer la contingence de la stratégie déployée par les entreprises et la configuration de leurs systèmes de contrôle de gestion (la gestion budgétaire, la décentralisation organisationnelle et les méthodes de calcul de coûts...). La relation entre stratégie et contrôle dans la mesure où la 
nature des systèmes et processus de contrôle ne peuvent pas être séparés de considérations stratégiques (Robert Simons, 1987). Trois types d’opérationnalisations de la stratégie dans les recherches contingentes en stratégie et contrôle ont été proposés par : le positionnement, la mission ou la typologie de Miles et Snow (1978). Langfield-Smith (1997) affirme que la stratégie n’était pas utilisée comme une variable dans la recherche sur les systèmes de contrôle avant les années quatre-vingts, cela est dû par l'inexistence jusqu'à la fin des années soixante-dix de typologies permettant d’opérationnaliser la variable stratégie comme une variable contingente (Chapman, 1997). La stratégie qui est un facteur de contingence tient une place croissante dans l'explication des systèmes de contrôle (Dent, 1990 ; Langfield-Smith, 1997 ; Bouquin, 2000).

De ces travaux, nous formulons l'hypothèse suivante :

Hypothèse 2 : "La planification stratégique est un préalable de l'adoption du contrôle de gestion ".

\subsection{La complexité de l'organisation et l'évolution des systèmes de contrôle de gestion}

La complexité, comprise comme « l’impossibilité d'une formalisation où tous les acteurs partie prenante d'un secteur trouveraient leur compte, c'est-à-dire finalement les réponses aux questions qu'ils se posent » (Moisdon et Tonneau, 1999). La complexité des organisations est un argument utilisé par Johnson et Kaplan pour fustiger les méthodes classiques de comptabilité analytique. Bruns et Waterhouse (1975) ont observé que les pratiques budgétaires les plus sophistiquées se retrouvent dans les structures les plus décentralisées. Ensuite, Merchant (1981) a montré que le processus budgétaire est plus formalisé, plus complexe, et plus participatif dans les organisations les plus décentralisées. Quelques années plus tard, Kalika (1987) a relevé que les organisations les plus différenciées et décentralisées au plan structurel disposent des systèmes de planification et de contrôle les plus développés.

La structure de gestion de l'hôpital (le découpage analytique) doit cohabiter avec une structure médicale liée au schéma de responsabilité médicale. Il n’y a pas toujours correspondance entre les niveaux de ces deux structures, ce qui rend l'appréhension et l'évaluation des activités encore une fois peu évidentes pour le contrôle de gestion. La complexité se manifeste plus particulièrement par le découpage de l'activité médicale (qui requiert des compétences multiples) qui nécessite un découpage analytique toujours plus fin. Il devient évident que le problème de la démarche gestionnaire va se trouver dans la représentation même des activités, de ce fait, l'impact pour le contrôle de gestion est important. Les outils mis en place se doivent donc 
d’être non seulement cohérents avec le contexte, mais aussi pertinents par rapport à l'utilisation que l'on veut en faire.

De ces travaux, nous formulons l'hypothèse suivante :

Hypothèse 3 : "Les organisations les plus différenciées et décentralisées au plan structurel disposent des pratiques du contrôle de gestion les plus développées ».

\subsection{Le contrôle externe et l'adoption du contrôle de gestion}

L'hôpital est confronté à une double contrainte, des exigences concernant leurs prestations et des contraintes budgétaires, cela entraine une forte demande d'outils de gestion (Moisdon, 1999) expliquant l'emprunt de certaines techniques ou démarches au monde de l'entreprise. En effet, l'hôpital, de par la particularité de son activité, ne satisfait pas à ces conditions de base (Ouchi, 1977). La mesure de la production constitue toujours une difficulté importante. Le contrôle budgétaire et les outils qui l'accompagnent apparaissent ainsi peu adaptés à un pilotage effectif de la performance des établissements hospitaliers. Il s'agit d'un contrôle aveugle (Olson, 1985), d'un rituel (Olsen, 1971 ; Ouchi, 1977), d'une action hypocrite (Brunsson, 1986), d'un rituel rhétorique (Pettersen, 1995). Ce contrôle se révèle peu opérationnel car les informations utilisées ne sont pas déclinables en actions par le centre opérationnel au sens de Mintzberg (1982). Les constats réalisés par ces différents auteurs se révèlent très pessimistes quant à l'efficacité du système traditionnel. Par contre, Bartoli (1997) résume les trois logiques envisageables de la performance dans le triangle de la performance : efficacité, efficience et budgétisation. Ces trois logiques relient les trois notions : résultats, moyens et objectifs. La logique de budgétisation est traditionnelle dans la sphère publique. Elle découle du droit budgétaire, visant principalement à vérifier l'utilisation des fonds publics. Elle a induit la mise en place des outils de contrôle de gestion utilisés par les entreprises avec trois caractéristiques : une forte logique financière et budgétaire, un objectif de reporting et un fonctionnement centralisé. Elle implique une notion de performance conduisant à un suivi de conformité. À l'hôpital, ces outils sont destinés à être utilisés par la direction générale pour le suivi budgétaire, avec un objectif de justification par rapport aux tutelles (technique et financière). Il s'agit d'un contrôle externe puisqu'il a pour objectif d'informer ces dernières de l'affectation des ressources. Le contrôle externe s'exerçant sur les organisations hospitalières provenait de différentes origines :

- le ministère de la santé et ses directions régionales ;

- le ministère de l'économie et des finances avec ses directeurs régionaux, les receveurs, les contrôleurs de l'état, et les trésoriers payeurs, pour suivre la comptabilité des engagements de dépenses et pour vérifier 
l'approvisionnement budgétaire. Les lois de décentralisation ont entraîné un partage entre secteur de la santé et les secteurs sociaux ce qui a favorisé la multiplicité des contrôleurs externes (les ministères, les tutelles et les diverses caisses). "Le contrôle externe, quelle que soit sa forme, est ici souvent motivé par un objectif d'apprentissage et/ou d'amélioration des performances » (Burlaud A., Zarlowski P., 2003).

«Le renforcement du contrôle externe est légitime, il conduit à améliorer les structures de gouvernance de l'entreprise, la transparence de son fonctionnement, améliore son efficacité économique comme son efficience au bénéfice d'un plus grand nombre de parties prenantes » (Burlaud A., Zarlowski P., 2003).

De ces travaux, nous formulons l'hypothèse suivante :

Hypothèse 4 : "Le renforcement du contrôle externe conduit à améliorer les structures de gouvernance de l'hôpital et la mise en place du contrôle de gestion ".

\subsection{Le système d'information et l'adoption du contrôle de gestion}

Les théoriciens de la diffusion des innovations en technologie d'information ont conclu par le biais d'études que les grandes entreprises disposant d'un large système d'information sont plus disposées à adopter les innovations en technologie d'information. Selon Bouquin et Pesqueux (1999), avec l'introduction de système d'information, le contrôleur de gestion fournisseur d'information est également un administrateur dans la mesure où il se concentre plus sur l'animation, la rénovation des systèmes ainsi que la réorganisation des procédures. Le contrôleur de gestion se trouve au cœur du processus informationnel de l'entreprise, il est donc confronté aux bouleversements liés à l'usage des Technologies d'Information et de Communication. Ces bouleversements ont induit une évolution majeure du rôle informateur du contrôleur de gestion. Le problème se focalise essentiellement sur l'aspect de façonner, traiter, sélectionner et commenter l'information, et non plus de la manière de produire ou de sortir les données (Löning et Pesqueux, 1998). Le système d'information constitue un enjeu de la pratique actuelle de la gestion des organisations. Les études concernant l'impact du système d'information sur les techniques de la fonction de contrôle de gestion sont encore en nombre très limité. Combes et Labrousse (1997) pensent que la mise en place d'un système d'information permet d'élaborer rapidement les indicateurs, d'en assurer la cohérence, de mettre à jour les tableaux de bord selon les périodicités parfois très courtes et de les transmettre de façon rapide aux différents acteurs de l'organisation. Le système d'information doit s'adapter à un ensemble de variables contingentes telles que la stratégie, la taille de l'organisation, et l'environnement (Chenhall, 2003 ; Santin et Van Caillie, 2008). Il a facilité 
le changement d'un travail habituel vers un travail plus analytique. Les contrôleurs ont plus de temps pour l'exécution des activités à valeur ajoutée reliées au contrôle de gestion et à la prise de décision (Scapens et Jazayeri, 2003 ; De Ronge, 2000).

De ces travaux, nous formulons l'hypothèse suivante :

Hypothèse 5 : "La mise en place d'un système d'information est un préalable de l'adoption du contrôle de gestion ».

\subsection{Le profil du contrôleur de gestion et l'évolution du contrôle de gestion}

Chiapello E. (1990) a montré que le contrôle de gestion traditionnel était plutôt l'apanage des anciens contrôleurs, tandis que le conseil était plutôt fréquent chez les jeunes diplômés, ce que la chercheuse interprète comme un rêve de jeune, auquel la lassitude met fin après de nombreuses années de pratique difficile, pour laisser la place au contrôle de gestion traditionnel. Le contrôle de gestion s'est développé au travers des actions d'établissement de normes et de prévisions financières, associées à un contrôle des réalisations. Dans la conception bureaucratique de la fonction du contrôleur de gestion, ce dernier était avant tout responsable de la définition du système de contrôle de gestion (définition de la nature et de la forme des comparaisons à effectuer...) et de la mise à disposition des décideurs des informations de gestion prédéfinies. Il faut souligner qu' « on ne peut envisager le rôle dévolu au contrôle de gestion, sans s'arrêter aux caractéristiques propres aux contrôleurs de gestion en place dans les entreprises. De même, un certain style de contrôle de gestion ne peut fonctionner, que si le contrôleur de gestion adhère à ce style et est apte à le faire fonctionner » (Fornerino M., Godener A., 2006).

Bollecker M. (2002) considère que la finalité de la fonction selon ce courant est «la surveillance des activités, principalement au service de la direction générale ». Des études ont montré que «le profil du contrôleur, nous permet d'affirmer qu'il a un rôle important dans la différenciation des systèmes de contrôle, ainsi, un profil technique a tendance à exercer un rôle diagnostic ou traditionnel, tandis qu'un profil gestionnaire est plus en interaction avec les opérationnels et donc, plus susceptible de « faire émerger des nouvelles opportunités stratégiques » (Ahsina K., 2013). Cet auteur affirme que "le profil gestionnaire des contrôleurs de gestion a tendance à favoriser le développement des systèmes interactifs de contrôle de gestion ».

De ces travaux, nous formulons l'hypothèse suivante :

Hypothèse 6 : "Le profil du responsable du contrôle de gestion influence la mise en place du contrôle de gestion ". 


\subsection{La mise en place du contrôle de gestion et son impact sur la performance organisationnelle}

La mise en place du contrôle de gestion a été depuis toujours accompagnée de la performance comme le souligne cette citation : le contrôle de gestion est « le processus par lequel les dirigeants s’assurent que les ressources sont obtenues et utilisées, avec efficience, efficacité et pertinence, conformément aux objectifs de l'organisation, et que les actions en cours vont bien dans le sens de la stratégie définie. Il correspond encore au processus qui garantit que les actions de la firme sont performantes, c'està-dire que la valeur des ressources employées reste bien inférieure à la valeur créée socialement reconnue par le marché » (Anthony, 1988). Gervais (2009), en complétant cette définition, a définit la notion de performance comme étant la combinaison de l'efficience (résultat atteint en rapport aux moyens engagés), de l'efficacité (capacité à atteindre les objectifs prévus et voulus) et de la pertinence (adéquation entre les objectifs et les moyens à disposition). Les travaux d'Ouchi (1979) sur le contrôle ont montré différentes modalités en fonction de la capacité à analyser et à avoir connaissance des facteurs de performance. En étudiant les modes de contrôle de l'organisation, Ouchi et Maguire (1975) suggèrent que le contrôle par la mesure et la gestion des outputs servent aux managers à obtenir des preuves objectives de performance. "Le contrôle de gestion traditionnel, qui se contente de mettre en évidence des écarts entre prévisions et réalisations, et s’intéresse exclusivement à l'aspect financier, ne participe pas efficacement aux efforts d'adaptation de la firme à son environnement » (Gervais M. et Thenet G., 1998). Plusieurs recherches se sont succédées depuis les années soixante-dix pour comprendre l'impact des pratiques du contrôle budgétaire sur la performance et sur de nombreux autres faits organisationnels (gestion à court terme, manipulation des résultats, stress...), mais l'explication de la forme prise par le contrôle budgétaire est restée moins étudiée. Une étude au Maroc a pu montrer que « la mise en place et le développement des systèmes de contrôle apportent une performance supérieure aux entreprises » (Ahsina K., 2013).

Le contrôle de gestion tourné vers l'avenir, est plus ouvert à des perspectives non exclusivement financières, et surtout plus adaptable aux besoins des managers. Il est susceptible d'aider ces derniers dans leurs décisions stratégiques ou quotidiennes.

De ces travaux, nous formulons l'hypothèse suivante :

Hypothèse 7 : "Les hôpitaux qui ont mis en place le contrôle de gestion sont plus performants que ceux qui en sont dépourvus ".

De la synthèse de cette revue de la littérature sur les déterminants de l'adoption du contrôle de gestion en milieu hospitalier, découle notre modèle théorique de recherche suivant : 


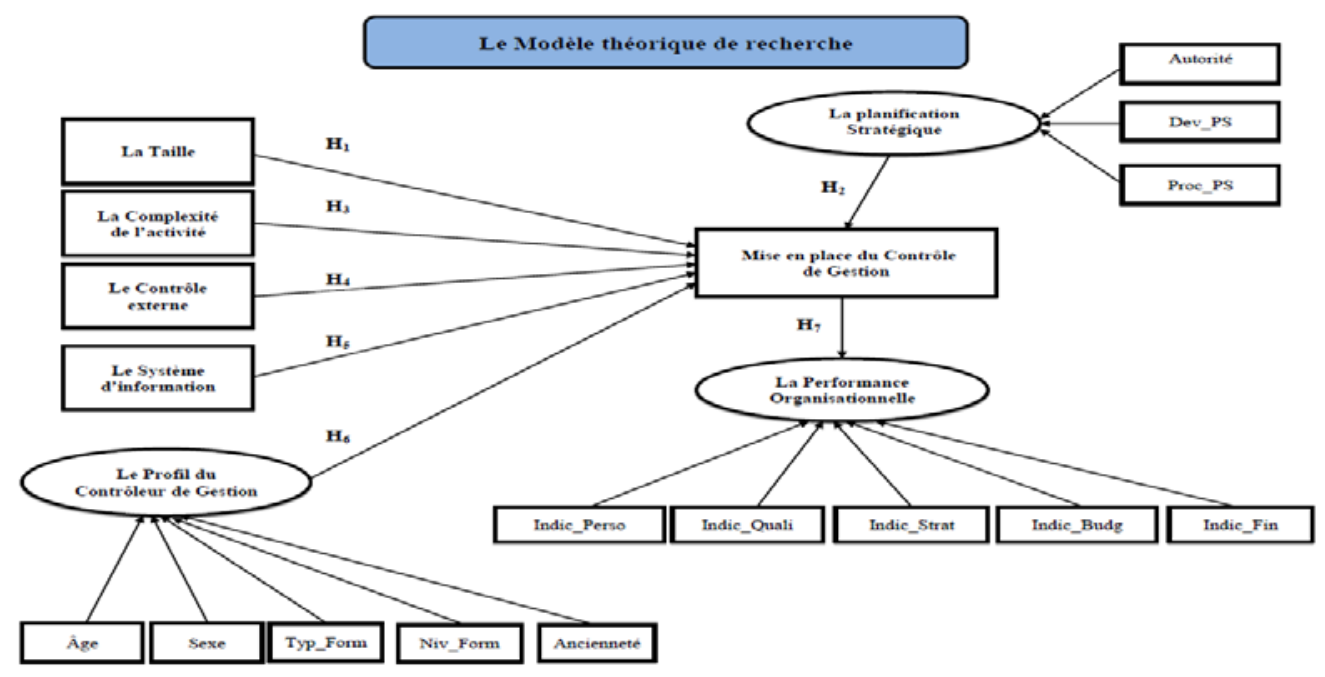

Source : établi par nous-mêmes

\section{Méthodologie de recherche :}

Notre démarche méthodologique est articulée autour de l'échantillonnage et des outils statistiques adoptés.

\subsection{Sélection de l'échantillon}

Notre échantillon est constitué de 52 hôpitaux Marocains. Nous avons mené une enquête par questionnaire qui comporte une diversité de questions et se subdivise en huit parties, afin de tester notre modèle. Ce questionnaire a été adressé aux responsables de contrôle de gestion et/ou responsables administratifs et financiers. Il a été envoyé, par voie postale et par e-mail, accompagné d'une lettre d'introduction dans laquelle l'optique de la recherche était précisée.

Tableau $n^{\circ} 1$ - Les hôpitaux de notre échantillon répartis suivant leur type

\begin{tabular}{|l|c|c|}
\hline \multicolumn{1}{|c|}{ Le type d'hôpital } & Effectif & Fréquence \\
\hline Hôpital public du CHU & 27 & $52 \%$ \\
\hline Hôpital public du CHR & 12 & $23 \%$ \\
\hline Hôpital public du CHP & 11 & $21 \%$ \\
\hline Hôpital Semi-public (HSP) & 2 & $4 \%$ \\
\hline Total & 52 & $100 \%$ \\
\hline
\end{tabular}

Source : établi par nous-mêmes

\subsection{Les outils statistiques adoptés}

Pour analyser l'incidence de l'adoption du contrôle de gestion par les hôpitaux publics, nous avons procédé par une analyse de données descriptives par le logiciel SPSS.v22. 


\section{Résultats :}

Dans cette section seront analysées les variables explicatives de notre modèle théorique de recherche.

\subsection{Existence du système de contrôle de gestion}

Tableau $\mathbf{n}^{\circ} 2$ - La répartition des hôpitaux selon l'existence du système de contrôle de gestion

\begin{tabular}{|c|c|c|}
\hline \multicolumn{1}{|c|}{ Existence du système de contrôle de gestion } & Effectif & Fréquence \\
\hline Existence du système de contrôle de gestion & 29 & $56 \%$ \\
\hline Absence du système de contrôle de gestion & 23 & $44 \%$ \\
\hline Total & 52 & $100 \%$ \\
\hline
\end{tabular}

Source : établi par nous-mêmes

La majorité (56\%) des hôpitaux possède un système de contrôle de gestion, pour le reste (44\%), l'absence du système de contrôle de gestion ne signifie pas que rien n'a été entrepris dans ce domaine. Le système de contrôle de gestion est supplanté par d'autres techniques de gestion (les tableaux de bord) ou encore que tous les préalables ne sont pas encore réunis.

\subsection{Analyse de la variable " planification stratégique "}

La planification stratégique va être appréhendée à travers l'étude des différentes variables relatives à :

\subsubsection{Le responsable de la planification stratégique}

Tableau $n^{\circ} 3$ - Le responsable de la planification stratégique

\begin{tabular}{|c|c|c|}
\hline Le responsable de la planification stratégique & Effectif & Fréquence \\
\hline Le directeur de l'hôpital & 42 & $81 \%$ \\
\hline Autres & 10 & $19 \%$ \\
\hline Total & 52 & $100 \%$ \\
\hline
\end{tabular}

Source : établi par nous-mêmes

La planification stratégique est sous l'autorité décisionnelle du directeur de l'hôpital avec $81 \%$, de ce fait, on peut confirmer qu'il est fortement impliqué dans la planification stratégique de l'ensemble des hôpitaux publics.

\subsubsection{Le processus de la planification stratégique}

Tableau $n^{\circ} 4$ - Le processus de la planification stratégique

\begin{tabular}{|l|c|c|}
\hline \multicolumn{1}{|c|}{ Le processus de la planification stratégique } & Effectif & Fréquence \\
\hline Programmation bi-annuelle & 1 & $2 \%$ \\
\hline Programmation annuelle & 11 & $21 \%$ \\
\hline Projet d'établissement hospitalier sur 5 ans (PEH) & 40 & $77 \%$ \\
\hline Total & 52 & $100 \%$ \\
\hline
\end{tabular}

Source : établi par nous-mêmes 
Le processus de la planification stratégique est conçu à 77\% par '’le projet d'établissement hospitalier sur 5 ans"', et à $21 \%$ par une programmation annuelle, alors que la programmation bi-annuelle ne représente que $2 \%$.

\subsection{Analyse de la variable " contrôle externe "}

Le contrôle externe va être appréhendé à travers l'étude des différentes variables relatives à :

\subsubsection{L'organisation du contrôle externe}

Tableau $n^{\circ} 5$ - L'organisation du contrôle externe

\begin{tabular}{|l|c|c|}
\hline L'organisation du contrôle externe & Effectif & Fréquence \\
\hline Le ministère de la santé & 34 & $65 \%$ \\
\hline La direction régionale de la santé & 13 & $25 \%$ \\
\hline La délégation de la santé & 0 & $0 \%$ \\
\hline Le directeur de l'hôpital & 0 & $0 \%$ \\
\hline Le conseil d'administration & 5 & $10 \%$ \\
\hline Le comité médical d'établissement & 0 & $0 \%$ \\
\hline Total & 52 & $100 \%$ \\
\hline
\end{tabular}

Source : établi par nous-mêmes

Les résultats de ce tableau nous confirment que le contrôle externe exercé sur les hôpitaux publics relève de la compétence du ministère de la santé (la tutelle) comme ce qui est dans la littérature, il est représenté par $65 \%$ des cas, il est exercé par la direction régionale de la santé avec $25 \%$, et à $10 \%$ par le conseil d'administration, par contre la délégation de la santé, le comité médical d'établissement et le directeur de l'hôpital n’y exercent aucun.

\subsection{Analyse de la variable « système d'information "}

Le système d'information va être appréhendé à travers l'étude de ces différentes variables :

\subsubsection{L'apport du système d'information au contrôle de gestion}

Tableau $n^{\circ} 6$ - L'apport du système d'information au contrôle de gestion

\begin{tabular}{|c|c|c|}
\hline L'apport du système d'information au CDG & Effectif & Fréquence \\
\hline La traçabilité & 13 & $25 \%$ \\
\hline La prise de décisions & 14 & $27 \%$ \\
\hline La mise à jour & 25 & $48 \%$ \\
\hline Total & 52 & $100 \%$ \\
\hline
\end{tabular}

Source : établi par nous-mêmes 
On observe que le système d’information permet au contrôle de gestion d'être à jour avec 48\% des réponses, de prendre des décisions avec $27 \%$ et de garder la traçabilité avec 25\%. Ce qui explique l’importance du système d’information pour le contrôle de gestion dans les hôpitaux publics.

\subsection{L’outil du contrôle de gestion prédominant}

Tableau $\mathbf{n}^{\circ} 7$ - L'outil du contrôle de gestion prédominant

\begin{tabular}{|c|c|c|}
\hline L'outil du contrôle de gestion prédominant & Effectif & Fréquence \\
\hline La comptabilité publique & 50 & $96 \%$ \\
\hline La comptabilité budgétaire & 0 & $0 \%$ \\
\hline La comptabilité générale & 2 & $4 \%$ \\
\hline La comptabilité analytique & 0 & $0 \%$ \\
\hline Total & 52 & $100 \%$ \\
\hline
\end{tabular}

Source : établi par nous-mêmes

Les résultats de ce tableau nous confirment que 96\% des hôpitaux utilisent la comptabilité publique comme outil du contrôle de gestion prédominant soit la totalité des hôpitaux publics, alors que seulement deux hôpitaux soit 4\% utilisent la comptabilité générale, c’est le cas des hôpitaux semi-publics.

\section{Conclusion :}

Cette recherche avait pour objectif de réaliser une revue de la littérature sur les déterminants de l'adoption du contrôle de gestion par les hôpitaux publics Marocains.

Il ressort de l'analyse des travaux antérieurs que la taille, la planification stratégique, la complexité de l’organisation, le contrôle externe, le système d'information et le profil du contrôleur, expliquent la mise en place du contrôle de gestion dans les entreprises en général et dans les hôpitaux publics en particulier.

Les résultats de l'analyse descriptive qu’on a réalisé, nous ont donné une vue générale sur certains outils utilisés par les hôpitaux publics Marocains. Une analyse plus approfondie nous permettra de valider notre modèle conceptuel et une meilleure congruence à la pratique réelle des hôpitaux Marocains.

\section{References :}

1. Ahsina, K. (2012). Management control systems and performance: Essay of Modelling. Kuwait Chapter of Arabian Journal of Business and Management Review. 1.11 (Jul 2012): 17-28.

2. Ahsina, K. (2013). Les conditions de réplicabilité des pratiques de contrôle de gestion en contexte Marocain: un essai de modélisation. 
Revue Marocaine de Recherche en Management et Marketing. 8. : 293-306

3. Ahsina, K. , Taouab O. , Nafzaoui M.A. (2014). Adoption et différenciation des systèmes de contrôle de gestion par les établissements publics Marocains: un essai de modélisation. European Scientific Journal, February 2014, Edition Vol.10, No.4.

4. Anthony, R. N. (1988). The management control function. The Harvard Business Press.

5. Anthony, R. N. (1965). Planning and control system a frame work for analysis. Boston, Harvard University Press.

6. Bollecker, M. (2002). Le rôle des contrôleurs de gestion dans l'apprentissage organisationnel: Une analyse de la phase de suivi des réalisations. Comptabilité-Contrôle-Audit 8(2): 109-126.

7. Bouquin, H. (2000). Contrôle et stratégie. Encyclopédie de comptabilité, contrôle de gestion et audit: 533-546.

8. Bouquin, H. and Y. Pesqueux (1999). Vingt ans de contrôle de gestion ou le passage d'une technique à une discipline. ComptabilitéContrôle-Audit 5(3): 93-105.

9. Bruns, W. J. and J. H. Waterhouse (1975). Budgetary control and organization structure. Journal of accounting research: 177-203.

10. Brunsson, N. (1986). Organizing for inconsistencies: On organizational conflict, depression and hypocrisy as substitutes for action. Scandinavian Journal of Management Studies 2(3-4): 165185.

11. Burlaud, A. et P. Zarlowski (2003). Le contrôle externe : quelles modalités pour quels enjeux ?. Revue française de gestion, 2003(6):147, page 18.

12. Chapman, C. S. (1997). Reflections on a Contingent View of Accounting. Accounting, Organizations and Society 22(2): 189-205.

13. Chenhall, R. H. (2003). Management control systems design within its organizational context: findings from contingency-based research and directions for the future. Accounting, Organizations and Society 28(2): 127-168.

14. Chiapello, E. (1990). Contrôleurs de gestion, comment concevez-vous votre fonction? Echanges 92(4): 7-36.

15. Combes, J.E., et al. (1997). Audit financier et contrôle de gestion. Publi-Union Paris.

16. Dahlgren, J. , Holmström, M. , Nehler, H. (2001). Activity-Based Costing - Diffusion and Adoption. Communication à la conférence annuelle de l'European Accounting Association, Athènes.

17. De Rongé, Y. (2000). Coûts. Encyclopédie de comptabilité, contrôle de gestion et audit, Economica. 
18. Dent, J. F. (1990). Strategy, organization and control: some possibilities for accounting research. Accounting, Organizations and Society 15(1-2): 3-25.

19. Dupuy, C. (1990). Village associations in Senegal: economic functions and financing arrangements. Revue Tiers Monde(122): 351-375.

20. Fornerino, M. and A. Godener (2006). Etre contrôleur de gestion en France aujourd'hui: conseiller, adapter les outils... et surveiller. Finance Contrôle Stratégie 9(1): 187-208.

21. Gervais, M. and G. Thenet (1998). Planification, gestion budgétaire et turbulence. Finance, contrôle, stratégie 1(3): 57-84.

22. Gervais, M. (2009). Contrôle de gestion des activités de service. in Encyclopédie de Comptabilité, Contrôle de gestion et Audit, $2^{\text {ème }}$ édition : 547-562.

23. Kalika, M. (1987). Structures d'entreprises, réalités, déterminants, performances. Économica.

24. Langfield-Smith, K. (1997). Management Control Systems and Strategy: a critical review. Accounting, Organizations and Society 22(2): 207-232.

25. Löning, H., et al. (1998). Le contrôle de gestion. Dunod Paris.

26. Merchant, K. A. (1981). The control function of management. Sloan management review 23(4): 43-55.

27. Miles, R. E., et al. (1978). Organizational strategy, structure, and process. Academy of management review 3(3): 546-562.

28. Mintzberg, H. (1982). Structures et dynamique des organisations. Paris: Éditions d’Organization.

29. Mintzberg, H. (2006). Filemanager au quotidien. Les 10 rôles du cadre.

30. Moisdon, J. C. and D. Tonneau (1999). La démarche gestionnaire à l'hôpital: Recherches sur la gestion interne. S. Arslan.

31. Moisdon, J. C. (1999). L'instrument multiprise. In Du mode d'existence des outils de gestion, Seli- Arslan : 114-133.

32. Nobre, T. (2001). Méthodes et outils du contrôle de gestion dans les PME. Finance, Contrôle, Stratégie, juin, 4(2): 119-148.

33. Olsen, J. P. (1971). Local Budgeting, Decision Making or Ritual Act. Scandinavian Political Studies, 5(3): 85-118.

34. Olson, O. (1985). Quasiplanning and Blind Control. Woeking paper, Dept of business administration, University of Gotenburg.

35. Ouchi, W. G. (1977). The relationship between organizational structure and organizational control. Administrative science quarterly: 95-113. 
36. Ouchi, W. G. (1979). A conceptual framework for the design of organizational control mechanisms. Readings in Accounting for Management Control, Springer: 63-82.

37. Ouchi, W. G. and M. A. Maguire (1975). Organizational control: Two functions. Administrative science quarterly: 559-569.

38. Pettersen, I. J. (1995). Budgetary control of hospitals-Ritual rhetorics and rationalized myths? Financial Accountability and Management 11: 207-207.

39. Porter, M. E. (1985). Competitive advantage: creating and sustaining superior performance. 1985. New York: Free Press.

40. Santin, S. and D. Van Caillie (2008). Le design du système de contrôle de gestion des PME: une quête de stabilité adaptative. La Comptabilité, le Contrôle et l'Audit entre Changement et Stabilité.

41. Scapens, R. W. and M. Jazayeri (2003). ERP systems and management accounting change: opportunities or impacts? A research note. European accounting review 12(1): 201-233.

42. Simons, R. (1987). Accounting Control Systems and Business Strategy: an empirical analysis. Accounting, Organizations and Society 12(4): 357-374. 